\title{
Coulisses
}

Revue de théâtre

25 | Hiver 2002

Varia

\section{Bilan du colloque : Thé@tre et (nouvelles) technologies}

\section{Lucile Garbagnati}

\section{(2) OpenEdition}

1 Journals

Édition électronique

URL : http://journals.openedition.org/coulisses/6173

DOI : $10.4000 /$ coulisses. 6173

ISSN : 2546-9460

Éditeur

Presses universitaires de Franche-Comté

\section{Édition imprimée}

Date de publication : 1 janvier 2002

Pagination : 144-145

ISBN : 2-84627-052-X

ISSN : 1150-594X

\section{Référence électronique}

Lucile Garbagnati, «Bilan du colloque : Thé@tre et (nouvelles) technologies », Coulisses [En ligne], 25 | Hiver 2002, mis en ligne le 24 octobre 2019, consulté le 24 septembre 2020. URL : http:// journals.openedition.org/coulisses/6173; DOI : https://doi.org/10.4000/coulisses.6173

Ce document a été généré automatiquement le 24 septembre 2020.

Coulisses 


\title{
Bilan du colloque : Thé@tre et (nouvelles) technologies
}

\author{
Lucile Garbagnati
}

Le colloque Théâtre et (nouvelles) technologies est l'acte IV d'un programme de recherches sur quatre ans mené par le centre de recherches Jacques-Petit et le Théâtre Universitaire de Franche-Comté. En mai 1998, le colloque Théâtre et Sciences ${ }^{1}$ avait été l'acte d'exposition d'une problématique générale; en juin 1999 Quel répertoire théâtral traitant de la science ${ }^{2}$ répertoriait les œuvres et les caractéristiques de ce de que l'on peut appeler un «théâtre de sciences ». En juin 2000, le colloque Temps scientifique, temps théâtral $^{3}$ a examiné l'expression et les modalités du temps en science et au théâtre.

2 Le colloque Théâtre et (nouvelles) technologies a invité à s'interroger au
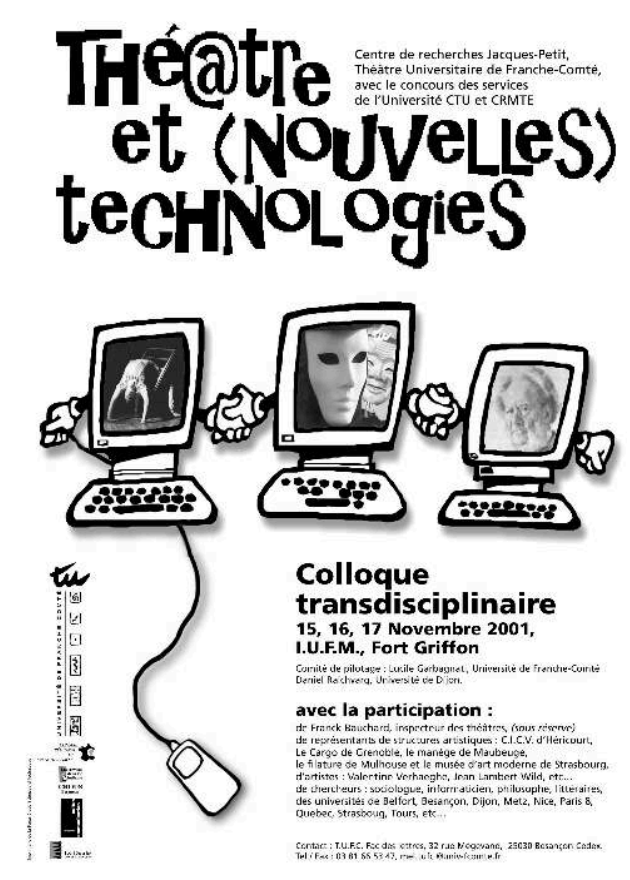
cours de 26 communications sur le rapport entre théâtre et technologies et plus particulièrement les «nouvelles technologies » selon quelques axes :

-Quels rapports le théâtre entretient-il avec les «nouvelles» technologies, quelle que soit l'époque considérée?

- Les nouvelles technologies modifient-elles l'écriture? Changent-elles la perception du spectateur? Dévoilent-elles des horizons nouveaux aux créateurs et/ou aux spectateurs ou bloquent-elles toute forme de créativité ? Sont-elles stimulantes ou suscitent-elles la passivité ? Qu'en est-il de l'interactivité ? 
- Sont-elles amenées à supplanter la création humaine? à remplacer le corps vivant par le corps virtuel?

3 En fait, il ne s'agit pas moins de la définition et de la fonction du théâtre, d'une interrogation sur la spécificité du spectacle vivant, de l'émergence de nouvelles formes d'art spectaculaire.

\section{Les participants}

4 Comme les précédents, le colloque a été transdisciplinaire. Il a rassemblé des artistes et des universitaires scientifiques et littéraires de toutes les disciplines, de diverses universités, entre autres de l'université de Québec. N'ayant pu se déplacer, elles avaient envisagé une vidéo conférence qu'il a fallu annuler au dernier moment pour cause de grève de techniciens au Québec !!! Il faut souligner le nombre de jeunes docteurs ou doctorants, ce qui tendrait à montrer l'intérêt de la question pour la génération montante. Des artistes reconnus, Jean Lambert-wild ou Valentine Verhaegue, et d'autres à des stades plus ou moins expérimentés ont exposé leur démarche et leur raison sous forme orale ou vidéo. Les trois plus grosses structures françaises spécialisées dans la question: le C.I.C.V. à Hérimoncourt, le Cargo à Grenoble, Le manège à Maubeuge ont développé leurs points de vue et leurs politiques. Il est toutefois regrettable que des organismes de diffusion de la culture scientifique n'aient pas été présents à titre d'intervenants ou d'auditeurs, pas plus d'ailleurs que les représentants des scènes labellisées. On ne peut donc que se réjouir de la participation active du service des nouvelles technologies de l'Université (C.T.U. et C.R.M.T.E.) qui montre l'intérêt de l'université pour la technologie mais pour les rapports qu'elle peut entretenir avec l'art.

\section{L'événement artistique}

5 Chaque colloque s'est caractérisé par un événement artistique de premier plan. Le colloque Théâtre et sciences avait accueilli pas moins de cinq spectacles, tous genres théâtraux confondus. Celui sur le répertoire pour un théâtre de sciences avait accueilli, quant à celui sur le temps il a eu le privilège de la lecture en première mondiale de la pièce de Gatti, tout juste finie d'écrire - Incertitudes de la mécanique quantique devenant chant des oiseaux du Graal pour l 'entrée des groupes (de Galois) dans le langage dramatique ${ }^{4}$. Celui sur les nouvelles technologies a bénéficié en première mondiale de la première pièce de théâtre conçue exclusivement pour internet, grâce au soutien de La Filature de Mulhouse, Côté noir, côté blanc qui a suscité beaucoup de questions et de commentaires passionnés.

6 Côté public, le colloque a été suivi par une cinquantaine de personnes dont une vingtaine très assidue. Une dizaine d'entre elles participent d'ailleurs aux travaux depuis le début, tantôt communicant, tantôt auditeur. La présence d'élèves de B.T.S. audiovisuels, extrêmement attentifs, pendant la demi-journée qu'ils ont suivie, a prouvé tout l'intérêt que suscitait la question.

7 Les Actes du colloque devraient paraître en juin au C.R.D.P. et mettre en évidence la richesse des communications et la nouveauté de la réflexion sur ce sujet 
exceptionnellement traitée à l'université, puisque, si l'on en croit Franck Bauchard, inspecteur des spectacles, c'était le premier du genre.

\section{Colloques et création d'un site internet, informatif et interactif}

Dans un terrain quasiment vierge, la recherche avait été programmée pour quatre ans de manière à faire, sinon le tour de la question, du moins poser un certain nombre de jalons. À l'issue du programme, la question se pose donc de savoir la suite à donner : arrêter ? continuer ce rendez-vous annuel ? tout en sachant que l'idée d'un festival de théâtre de sciences est toujours sous-jacente. Toutefois, elle doit être renvoyée au plus tôt en 2004. En effet les mandats des directeurs des salles bisontines s'arrêtent en 2001. Il faut attendre la nomination de leurs successeurs qui programmeront les prochaines saisons. À l'unanimité, les participants, anciens et nouveaux, ont souhaité continuer la réflexion commune. En effet, il y a peu de lieux consacrés à ce sujet et la forme de colloque permet non seulement de confronter des idées mais aussi de créer des relations suivies. Des rendez-vous plus fréquents sous forme de journée d'études ont été suggérés, ce qui implique des frais supplémentaires et une plus grande disponibilité. Une proposition s'est alors imposée : la création d'un site internet, informatif et interactif, avec des rencontres bisannuelles pour tester le virtuel à l'aune du vivant. Le projet est à l'étude et devrait se mettre en place courant 2002. Les structures théâtrales et universitaires présentes se sont d'entrée porté partie prenante du projet, la principale difficulté étant celle du webmaster. Les efforts de cette année se porteront donc sur la création du site, que nous tenterons d'évaluer en 2003.

\section{NOTES}

1. Actes, Presses du Centre UNESCO de Besançon diffusion SODIS, nov. 1998

2. Actes, L'Harmattan 2000

3. Actes, C.R.D.P. 2001, a obtenu le label Arts et culture

4. Publication, Les cahiers de Coulisses $\mathrm{n}^{\circ} 2$ 